\title{
Studies on Variability, Heritability, Correlation and Path Analysis for Yield, Yield Attributes in Rice (Oryza sativa L.)
}

\author{
B. Edukondalu ${ }^{1 *}$, V. Ram Reddy ${ }^{1}$, T. Shobha Rani ${ }^{1}$, \\ Ch. Aruna Kumari ${ }^{1}$ and B. Soundharya ${ }^{2}$ \\ ${ }^{1}$ Department of Genetics and Plant Breeding, College of Agriculture (PJTSAU), \\ Jagtial -505 529, Telangana, India \\ ${ }^{2}$ Department of Genetics and Plant Breeding, College of Agriculture (PJTSAU), \\ Rajendranagar, Hyderabad, 500-030, Telangana, India \\ *Corresponding author
}

\begin{tabular}{|c|c|}
\hline & A B S T R A C T \\
\hline & \multirow{7}{*}{$\begin{array}{l}\text { An investigation was carried out to study the heritability, correlation and path analysis for } \\
15 \text { characters in } 40 \text { genotypes of rice during Kharif, } 2016 \text {. Analysis of variance revealed } \\
\text { that mean sum of squares due to genotypes showed significant differences for all } 15 \\
\text { characters studied. Analysis of variability parameters revealed that the phenotypic } \\
\text { coefficient variation were higher than genotypic coefficient variation. The magnitude of } \\
\text { PCV and GCV was high for number of grains per panicle. High heritability coupled with } \\
\text { high genetic advance as per cent of mean was observed for number of grains per panicle, } \\
\text { number of tillers per plant, } 1000 \text {-grain weight, L/B ratio, plant height, kernel length, grain } \\
\text { yield per plant, kernel breadth and days to flowering. In general, magnitudes of genotypic } \\
\text { correlation were found to be higher than phenotypic correlations. The results indicated that } \\
\text { grain yield per plant showed significant positive association with number of tillers per } \\
\text { plant, panicle length and milling percentage. Path coefficient analysis revealed that number } \\
\text { of tillers per plant exerted the highest positive direct effect on grain yield per plant } \\
\text { followed by kernel breadth, L/B ratio, panicle length, milling percentage, days to } \\
\text { flowering and head rice recovery percentage. }\end{array}$} \\
\hline Keywords & \\
\hline & \\
\hline & \\
\hline Article Info & \\
\hline $\begin{array}{l}\text { Accepte } \\
21 \text { Sept } \\
\text { Availab } \\
10 \text { Octo }\end{array}$ & \\
\hline & \\
\hline
\end{tabular}

\section{Introduction}

Rice (Oryza sativa L.) is one of the most predominant food crops in India in terms of area, production and consumer preference. India is the second largest producer and consumer of rice in the world.

Rice is the premier food crop in India occupying nearly 43.0 million hectares with annual production of 106.54 million tonnes and productivity of $2424 \mathrm{Kg} \mathrm{ha}^{-1}$ per hectare (Indiastat, 2015-2016). Area under rice cultivation Telangana State in nearly 1.65 million hectares while the production is 4.3 million tonnes and productivity in $3162 \mathrm{~kg} \mathrm{ha}^{-1}$.

To meet the demands of growing population and to achieve food security in the country the present production levels need to be increased by two million tonnes every year.

It is estimated that 120 million tonnes of rice is required to feed the increasing population 
by 2020 . The selection criteria may be yield or one or more of the yield component characters. However, breeding for high yield crops require information on nature and magnitude of variation in the available material, relationship of yield with other agronomic characters and the degree of environmental influence on the expression of these component characters. Since grain yield in rice is quantitative in nature and polygenically controlled, effective yield improvement and simultaneous improvement of yield components are imperative. To enhance the yield productivity, genetic parameters and correlation studies between yield and yield components are per requisite to plan a meaningful breeding programme to develop high yielding inbreds and hybrids.

\section{Materials and Methods}

All the 40 genotypes were sown separately in the nursery on raised beds. Twenty five days old seedlings of each genotype were transplanted in two rows each in a $4 \mathrm{~m}$ length plot by adopting a spacing of $20 \mathrm{~cm}$ between rows and $15 \mathrm{~cm}$ between plants in a Randomized Block Design replicated twice during Kharif, 2016 at Regional Agricultural Research Station, Polasa, Jagtial, and Telangana State. Five plants of each genotype in each replication were selected are used to record data on plant height $(\mathrm{cm})$, number of tillers per plant, panicle length $(\mathrm{cm})$, number of grains per panicle, kernel length $(\mathrm{mm})$, kernel breadth $(\mathrm{mm})$, L/B ratio, 1000-grain weight $(\mathrm{g})$, hulling percentage, milling percentage, head rice recovery percentage, grain yield per plant (g) and bran oil percentage. The mean values were considered for statistical analysis.

Analysis of variance was done for partitioning the total variation due to treatments and replications according to procedure given by Panse and Sukhatme (1985). Correlation coefficients were determined as described by Singh and Chaudhary (1979).

\section{Results and Discussion}

Analysis of variance revealed significant differences for all 15 quantitative traits studied which was presented in Table 1. A perusal of genetic parameters revealed that phenotypic and genotypic coefficients of variation were high for number of grains per panicle, followed by number of tillers per plant, 1000-grain weight, L/B ratio, plant height, kernel breadth, kernel length and grain yield per plant were moderate. The values of genotypic and phenotypic coefficients of variation were low for days to maturity, days to flowering, panicle length, hulling percentage, milling percentage and head rice recovery. These results are in accordance with findings of Nagajyothi (2001) and Tara Satyavathi et al., (2001), Anis et al., (2016) for moderate GCV and PCV, Dhurai et al., (2014) and Ganapathi et al., (2014), Anis et al., (2016), Rukmini Devi et al., (2016) for high heritability and Dhurai et al.,(2014), Ganapati et al.,(2014) and Sachan (2015), Ananth kumar and Verma (2016) and Rukmini Devi et al., (2016).

High heritability coupled with high genetic advance as per cent of mean was observed for number of grains per panicle, number of tillers per plant, 1000-grain weight, L/B ratio, plant height, kernel length, grain yield per plant, kernel breadth and days to flowering indicated that these traits were controlled by additive type of gene action in the inheritance of these characters. These characters can be further improved by following simple selection procedure. The high estimates of heritability coupled with low genetic advance as percent of mean for milling percentage and hulling percentage indicated the presence of nonadditive gene effects, in addition to influence of environment to some extent (Table 2). 
Table.2 Magnitude of variability, heritability and genetic advance for yield and quality traits in rice (Oryza sativa L.)

\begin{tabular}{|c|c|c|c|c|c|c|c|c|}
\hline \multirow[b]{2}{*}{ S.no. } & \multirow[b]{2}{*}{ Character } & \multirow[b]{2}{*}{$\begin{array}{c}\text { General } \\
\text { Mean }\end{array}$} & \multicolumn{2}{|c|}{ Range } & \multirow{2}{*}{$\begin{array}{c}\text { Phenotypic } \\
\text { Coefficient } \\
\text { of } \\
\text { Variation } \\
\text { (PCV) }\end{array}$} & \multirow{2}{*}{$\begin{array}{c}\text { Genotypic } \\
\text { Coefficient } \\
\text { of } \\
\text { Variation } \\
\text { (GCV) }\end{array}$} & \multirow{2}{*}{$\begin{array}{c}\text { Heritability in } \\
\text { broad sense } \\
(\%) \\
\left(H_{b s}\right)\end{array}$} & \multirow[b]{2}{*}{$\begin{array}{c}\text { GA as per cent of } \\
\text { mean }(5 \%) \\
(\text { GAM) }\end{array}$} \\
\hline & & & Maximum & Minimum & & & & \\
\hline 1 & Days to flowering & 86.20 & 118.50 & 74.50 & 9.99 & 9.96 & 99.0 & 20.44 \\
\hline 2 & Days to maturity & 120.65 & 149.50 & 109.50 & 6.28 & 6.20 & 97.0 & 12.63 \\
\hline 3 & Plant height $(\mathrm{cm})$ & 101.02 & 151.00 & 77.60 & 12.17 & 12.10 & 98.0 & 24.79 \\
\hline 4 & Number of tillers/plant & 12.56 & 16.70 & 7.70 & 17.73 & 17.32 & 95.0 & 34.87 \\
\hline 5 & Panicle length $(\mathrm{cm})$ & 24.30 & 27.90 & 17.50 & 8.07 & 7.54 & 87.0 & 14.52 \\
\hline 6 & Number of grains / panicle & 143.25 & 333.80 & 76.50 & 44.01 & 43.32 & 96.0 & 87.85 \\
\hline 7 & Kernel length (mm) & 6.57 & 8.25 & 5.15 & 10.90 & 10.85 & 99.0 & 22.27 \\
\hline 8 & Kernel breadth (mm) & 1.92 & 2.70 & 1.65 & 11.82 & 11.21 & 90.0 & 21.91 \\
\hline 9 & L/B Ratio & 3.55 & 4.39 & 2.06 & 13.78 & 13.31 & 93.0 & 26.48 \\
\hline 10 & Grain yield per plant (g) & 24.90 & 29.80 & 19.00 & 11.26 & 10.99 & 95.0 & 22.08 \\
\hline 11 & 1000-grain weight (g) & 19.67 & 25.75 & 12.76 & 16.42 & 16.01 & 95.0 & 32.15 \\
\hline 12 & Hulling \% & 83.62 & 89.76 & 75.10 & 3.81 & 3.73 & 95.0 & 7.54 \\
\hline 13 & Milling \% & 75.81 & 80.89 & 68.90 & 4.69 & 4.60 & 96.0 & 9.28 \\
\hline 14 & Head rice recovery $\%$ & 64.04 & 71.88 & 51.72 & 7.74 & 7.51 & 94.0 & 15.00 \\
\hline 15 & Bran oil \% & 14.95 & 19.35 & 11.87 & 12.15 & 11.77 & 93.0 & 23.48 \\
\hline
\end{tabular}


Table.3 Phenotypic (P) and Genotypic (G) correlation coefficients of yield and quality traits in rice (Oryza sativa L.)

\begin{tabular}{|c|c|c|c|c|c|c|c|c|c|c|c|c|c|c|c|c|}
\hline Character & & DF & DM & PTH & No.TPP & $\mathbf{P L}$ & No.GPP & $\mathbf{K L}$ & KB & L/B & $1000 \mathrm{GW}$ & HUL & MIL & HRR & B O & GYP \\
\hline \multirow{2}{*}{ DF } & G & 1.0000 & 0.8531 & 0.2972 & 0.1666 & 0.2129 & 0.1938 & 0.0788 & -0.0402 & 0.0743 & -0.1483 & 0.1477 & -0.2778 & -0.0619 & -0.0701 & 0.1767 \\
\hline & $\mathbf{P}$ & 1.0000 & $0.8451^{* * *}$ & $0.2951 * *$ & 0.1608 & 0.1934 & 0.1924 & 0.0788 & -0.0317 & 0.0669 & -0.1430 & 0.1440 & $-0.2684^{*}$ & -0.0635 & -0.0651 & 0.1736 \\
\hline \multirow{2}{*}{ DM } & G & & 1.0000 & 0.1246 & 0.0985 & 0.0076 & 0.1865 & $\begin{array}{l}-0.0449 \\
\end{array}$ & 0.0113 & $\begin{array}{l}-0.0287 \\
\end{array}$ & $\begin{array}{l}-0.1564 \\
\end{array}$ & 0.0887 & -0.1719 & $\begin{array}{l}-0.0690 \\
\end{array}$ & -0.0280 & 0.1237 \\
\hline & $\mathbf{P}$ & & 1.0000 & 0.1232 & 0.0966 & 0.0028 & 0.1848 & -0.0426 & 0.0179 & -0.0325 & -0.1554 & 0.0779 & $\begin{array}{l}-0.1601 \\
\end{array}$ & $\begin{array}{l}-0.0639 \\
\end{array}$ & -0.0210 & 0.1238 \\
\hline \multirow{2}{*}{ PTH } & G & & & 1.0000 & 0.2775 & 0.4546 & -0.0231 & 0.2422 & 0.2985 & -0.0230 & 0.0823 & -0.0793 & -0.1485 & 0.2794 & 0.3655 & 0.2357 \\
\hline & $\mathbf{P}$ & & & 1.0000 & $0.2653^{*}$ & $0.4355^{* * *}$ & 0.0239 & $0.2368^{*}$ & $0.2858^{*}$ & -0.0267 & 0.0860 & -0.0817 & -0.1469 & $0.2688^{*}$ & $0.3572 * *$ & 0.2393 \\
\hline \multirow{2}{*}{ No.TPP } & G & & & & 1.0000 & 0.3561 & 0.0996 & 0.1835 & -0.1770 & 0.2068 & 0.0396 & 0.4170 & 0.2906 & -0.0850 & -0.2667 & $0.9649 * *$ \\
\hline & $\mathbf{P}$ & & & & 1.0000 & $0.3123^{* * *}$ & 0.0918 & 0.1771 & -0.1727 & 0.2013 & 0.0260 & $0.3931^{* *}$ & $0.2932^{* * *}$ & -0.0845 & $-0.2464 *$ & $0.9191 * *$ \\
\hline \multirow{2}{*}{ PL } & G & & & & & 1.0000 & -0.0679 & 0.3510 & -0.0323 & 0.1881 & 0.3589 & 0.2266 & 0.1030 & -0.0702 & -0.3284 & $0.3384^{* *}$ \\
\hline & $\mathbf{P}$ & & & & & 1.0000 & -0.0684 & $0.3196^{* * *}$ & -0.0317 & 0.1688 & $0.3339^{* * *}$ & $0.2242 *$ & 0.0760 & -0.0382 & $-0.2674^{*}$ & $0.3339 * *$ \\
\hline \multirow{2}{*}{ No.GPP } & G & & & & & & 1.0000 & -0.2368 & -0.4864 & 0.1610 & -0.6603 & 0.0282 & 0.3523 & 0.1597 & 0.1976 & 0.0845 \\
\hline & $\mathbf{P}$ & & & & & & 1.0000 & $-0.2283^{*}$ & $-0.4613^{* *}$ & 0.1625 & $0.6381^{\text {*** }}$ & 0.0299 & $0.3477^{* * *}$ & 0.1478 & 0.1852 & 0.0805 \\
\hline \multirow{2}{*}{$\mathbf{K L}$} & G & & & & & & & 1.0000 & 0.0573 & 0.7232 & 0.2996 & 0.0165 & -0.0744 & -0.0610 & -0.0538 & 0.1385 \\
\hline & $\mathbf{P}$ & & & & & & & 1.0000 & 0.0606 & $0.6960^{* *}$ & 0.2897 ** & 0.0100 & -0.0738 & -0.0630 & -0.0579 & 0.1314 \\
\hline \multirow{2}{*}{ KB } & G & & & & & & & & 1.0000 & -0.6296 & 0.6250 & -0.1782 & -0.4092 & 0.0657 & 0.2154 & -0.1847 \\
\hline & $\mathbf{P}$ & & & & & & & & 1.0000 & $-0.6542 * *$ & $0.5686^{* * *}$ & -0.1773 & $-0.3827 * *$ & 0.0635 & 0.1885 & -0.1462 \\
\hline \multirow{2}{*}{$\mathbf{L} / \mathbf{B}$} & G & & & & & & & & & 1.0000 & -0.2555 & 0.0887 & 0.1958 & -0.0601 & -0.1172 & 0.1819 \\
\hline & $\mathbf{P}$ & & & & & & & & & 1.0000 & $-0.2352^{*}$ & 0.0864 & 0.1851 & -0.0641 & -0.1042 & 0.1481 \\
\hline \multirow{2}{*}{1000 GW } & G & & & & & & & & & & 1.0000 & 0.0292 & -0.1519 & -0.2670 & -0.3288 & 0.0324 \\
\hline & $\mathbf{P}$ & & & & & & & & & & 1.0000 & 0.0308 & -0.1564 & $-0.2573^{*}$ & $-0.3022^{* *}$ & 0.0254 \\
\hline \multirow{2}{*}{ HUL } & G & & & & & & & & & & & 1.0000 & 0.1053 & -0.4396 & -0.1180 & $0.2873^{* * *}$ \\
\hline & $\mathbf{P}$ & & & & & & & & & & & 1.0000 & 0.1020 & $-0.4062 * *$ & -0.1049 & 0.2707 \\
\hline \multirow{2}{*}{ MIL } & G & & & & & & & & & & & & 1.0000 & 0.0157 & -0.0890 & $0.3254^{* * *}$ \\
\hline & $\mathbf{P}$ & & & & & & & & & & & & 1.0000 & 0.0091 & -0.0851 & $0.3214 * *$ \\
\hline \multirow{2}{*}{ HRR } & G & & & & & & & & & & & & & 1.0000 & 0.3154 & -0.0035 \\
\hline & $\mathbf{P}$ & & & & & & & & & & & & & 1.0000 & $0.3042^{* * *}$ & 0.0040 \\
\hline \multirow{2}{*}{ В O } & G & & & & & & & & & & & & & & 1.0000 & $-0.2867 * *$ \\
\hline & $\mathbf{P}$ & & & & & & & & & & & & & & 1.0000 & $-0.2787 * *$ \\
\hline \multicolumn{17}{|c|}{ * Significant at 5 per cent level; ** Significant at 1 per cent level } \\
\hline
\end{tabular}

DF: Days to flowering, DM: Days to maturity, PTH: Plant height $(\mathrm{cm})$, No.TPP: Number of tillers per plant, PL: Panicle length (cm), No.GPP: Number of grains per panicle, KL: Kernel length (mm), KB: Kernel breadth (mm), L/B: L/B Ratio, 1000 GW: 1000-grain weight, HUL: Hulling \%, MIL: Milling \%, HRR: Head rice recovery \%, B O: Bran oil \% GYP: Grain yield per plant (g). 
Table.4 Phenotypic (P) and Genotypic $(\mathrm{G})$ path coefficients of yield and quality traits in rice (Oryza sativa L.)

\begin{tabular}{|c|c|c|c|c|c|c|c|c|c|c|c|c|c|c|c|c|}
\hline Character & & DF & DM & PTH & No.TPP & $\mathbf{P L}$ & No.GPP & KL & KB & $\mathbf{L} / \mathbf{B}$ & $1000 \mathrm{GW}$ & HUL & MIL & HRR & B O & GYP \\
\hline \multirow{2}{*}{ DFF } & G & 0.1639 & -0.0438 & -0.0606 & $\begin{array}{l}0.1807 \\
\end{array}$ & 0.0319 & $\begin{array}{l}-0.0191 \\
\end{array}$ & -0.0426 & -0.0151 & 0.0432 & -0.0062 & -0.0266 & -0.0189 & -0.0035 & -0.0066 & 0.1767 \\
\hline & $\mathbf{P}$ & 0.0834 & $\begin{array}{l}-0.0003 \\
\end{array}$ & -0.0289 & $\begin{array}{l}0.1518 \\
\end{array}$ & 0.0236 & -0.0121 & $\begin{array}{l}-0.0117 \\
\end{array}$ & -0.0052 & 0.0088 & 0.0074 & -0.0158 & -0.0249 & -0.0043 & 0.0017 & 0.1736 \\
\hline \multirow[b]{2}{*}{ DM } & G & 0.1398 & -0.0514 & -0.0254 & 0.1068 & 0.0011 & -0.0184 & 0.0243 & 0.0042 & -0.0167 & -0.0065 & -0.0160 & -0.0117 & -0.0039 & -0.0026 & 0.1237 \\
\hline & $\mathbf{P}$ & 0.0704 & -0.0004 & -0.0121 & 0.0912 & 0.0003 & -0.0116 & 0.0063 & 0.0029 & -0.0043 & 0.0081 & -0.0086 & -0.0146 & -0.0043 & 0.0006 & 0.1238 \\
\hline \multirow{2}{*}{ PTH } & G & 0.0487 & -0.0064 & -0.2039 & 0.3010 & 0.0682 & 0.0023 & -0.1309 & 0.1120 & -0.0134 & 0.0034 & 0.0143 & -0.0101 & 0.0159 & 0.0346 & 0.2357 \\
\hline & $\mathbf{P}$ & 0.0246 & 0.0000 & $-\mathbf{- 0 . 0 9 8 0}$ & 0.2505 & 0.0532 & 0.0015 & -0.0351 & 0.0468 & -0.0035 & -0.0045 & 0.0090 & -0.0136 & 0.0181 & -0.0096 & 0.2393 \\
\hline \multirow{2}{*}{ No.TPP } & G & 0.0273 & -0.0051 & -0.0566 & 1.0847 & 0.0534 & -0.0098 & -0.0992 & -0.0664 & 0.1203 & 0.0016 & -0.0751 & 0.0198 & -0.0048 & -0.0252 & $0.9649 * *$ \\
\hline & $\mathbf{P}$ & 0.0134 & 0.0000 & -0.0260 & 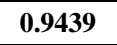 & 0.0381 & -0.0058 & -0.0262 & -0.0283 & 0.0264 & -0.0013 & -0.0433 & 0.0272 & -0.0057 & 0.0066 & $0.9191 * *$ \\
\hline \multirow{2}{*}{ PL } & G & 0.0349 & -0.0004 & -0.0927 & 0.3863 & 0.1500 & 0.0067 & -0.1897 & -0.0121 & 0.1095 & 0.0149 & -0.0408 & 0.0070 & -0.0040 & -0.0311 & $0.3384 * *$ \\
\hline & $\mathbf{P}$ & 0.0161 & 0.0000 & -0.0427 & 0.2947 & 0.1222 & 0.0043 & $\begin{array}{l}-0.0474 \\
\end{array}$ & -0.0052 & 0.0222 & -0.0173 & -0.0247 & 0.0070 & -0.0026 & 0.0072 & $0.3339^{* *}$ \\
\hline \multirow{2}{*}{ No.GPP } & G & 0.0318 & -0.0096 & 0.0047 & 0.1080 & -0.0102 & -0.0987 & 0.1280 & -0.1825 & 0.0937 & -0.0274 & -0.0051 & 0.0240 & 0.0091 & 0.0187 & 0.0845 \\
\hline & $\mathbf{P}$ & 0.0160 & -0.0001 & 0.0023 & 0.0867 & -0.0084 & -0.0628 & 0.0338 & -0.0755 & 0.0213 & 0.0331 & -0.0033 & 0.0323 & 0.0099 & -0.0050 & 0.0805 \\
\hline \multirow{2}{*}{ KL } & G & 0.0129 & 0.0023 & -0.0494 & 0.1990 & 0.0526 & 0.0234 & $\begin{array}{l}-0.5405 \\
\end{array}$ & 0.0215 & 0.4207 & 0.0124 & -0.0030 & -0.0051 & -0.0035 & -0.0051 & 0.1385 \\
\hline & $\mathbf{P}$ & 0.0066 & 0.0000 & -0.0232 & 0.1672 & 0.0390 & 0.0143 & $\begin{array}{l}-0.1482 \\
\end{array}$ & 0.0099 & 0.0914 & -0.0150 & -0.0011 & -0.0068 & -0.0042 & 0.0016 & 0.1314 \\
\hline \multirow{2}{*}{ KB } & G & -0.0066 & -0.0006 & -0.0609 & -0.1920 & -0.0048 & 0.0480 & -0.0310 & 0.3752 & -0.3663 & 0.0259 & 0.0321 & -0.0279 & 0.0037 & 0.0204 & -0.1847 \\
\hline & $\mathbf{P}$ & -0.0026 & 0.0000 & -0.0280 & -0.1631 & -0.0039 & 0.0290 & -0.0090 & 0.1636 & -0.0859 & -0.0295 & 0.0195 & -0.0355 & 0.0043 & -0.0051 & -0.1462 \\
\hline \multirow{2}{*}{$\mathbf{L} / \mathbf{B}$} & G & 0.0122 & 0.0015 & 0.0047 & 0.2243 & 0.0282 & -0.0159 & -0.3909 & -0.2362 & 0.5818 & -0.0106 & -0.0160 & 0.0133 & -0.0034 & -0.0111 & 0.1819 \\
\hline & $\mathbf{P}$ & 0.0056 & 0.0000 & 0.0026 & 0.1900 & 0.0206 & -0.0102 & -0.1031 & -0.1070 & 0.1313 & 0.0122 & -0.0095 & 0.0172 & -0.0043 & 0.0028 & 0.1481 \\
\hline \multirow{2}{*}{$1000 \mathrm{GW}$} & G & -0.0243 & 0.0080 & -0.0168 & 0.0430 & 0.0538 & 0.0652 & $\begin{array}{l}-0.1619 \\
\end{array}$ & 0.2345 & -0.1486 & 0.0415 & -0.0053 & -0.0103 & -0.0552 & -0.0311 & 0.0324 \\
\hline & $\mathbf{P}$ & -0.0119 & 0.0001 & -0.0084 & 0.0245 & 0.0408 & 0.0401 & -0.0429 & 0.0930 & -0.0309 & -0.0519 & -0.0034 & -0.0145 & -0.0173 & 0.0081 & 0.0254 \\
\hline \multirow{2}{*}{ HUL } & G & 0.0242 & -0.0046 & 0.0162 & 0.4524 & 0.0340 & -0.0028 & -0.0089 & -0.0669 & 0.0516 & 0.0012 & 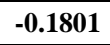 & 0.0072 & -0.0250 & -0.0112 & $0.2873 * *$ \\
\hline & $\mathbf{P}$ & 0.0120 & 0.0000 & 0.0080 & 0.3710 & 0.0274 & -0.0019 & -0.0015 & -0.0290 & 0.0113 & $\begin{array}{l}-0.0016 \\
\end{array}$ & $\begin{array}{l}-0.1101 \\
\end{array}$ & 0.0095 & -0.0273 & 0.0028 & 0.2707 \\
\hline \multirow{2}{*}{ MIL } & G & -0.0455 & 0.0088 & 0.0303 & 0.3152 & 0.0154 & -0.0348 & 0.0402 & -0.1535 & 0.1139 & -0.0063 & -0.0190 & 0.0681 & 0.0009 & -0.0084 & $0.3254^{* *}$ \\
\hline & $\mathbf{P}$ & -0.0224 & 0.0001 & 0.0144 & 0.2768 & 0.0093 & -0.0218 & 0.0109 & -0.0626 & 0.0243 & 0.0081 & -0.0112 & 0.0928 & 0.0006 & 0.0023 & $0.3214^{* *}$ \\
\hline \multirow{2}{*}{ HRR } & G & -0.0101 & 0.0035 & -0.0570 & -0.0922 & -0.0105 & -0.0158 & 0.0330 & 0.0246 & -0.0349 & -0.0111 & 0.0792 & 0.0011 & 0.0568 & 0.0299 & -0.0035 \\
\hline & $\mathbf{P}$ & -0.0053 & 0.0000 & -0.0263 & -0.0797 & -0.0047 & -0.0093 & 0.0093 & 0.0104 & -0.0084 & 0.0133 & 0.0447 & 0.0008 & 0.0672 & -0.0082 & 0.0040 \\
\hline \multirow{2}{*}{ В O } & G & -0.0115 & 0.0014 & -0.0745 & -0.2892 & -0.0493 & -0.0195 & 0.0291 & 0.0808 & -0.0682 & -0.0136 & 0.0213 & -0.0061 & 0.0179 & 0.0947 & $-0.2867 * *$ \\
\hline & $\mathbf{P}$ & -0.0054 & 0.0000 & -0.0350 & -0.2326 & -0.0327 & -0.0116 & 0.0086 & 0.0308 & -0.0137 & 0.0157 & 0.0115 & -0.0079 & 0.0204 & -0.0269 & $-0.2787^{* *}$ \\
\hline & & & & Genotyn & & & 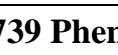 & & & & & & & & & \\
\hline
\end{tabular}

* Significant at 5 per cent level; ** Significant at 1 per cent level

DF: Days to flowering, DM: Days to maturity, PTH: Plant height $(\mathrm{cm})$, No.TPP: Number of tillers per plant, PL: Panicle length (cm), No.GPP: Number of grains per panicle, KL: Kernel length (mm), KB: Kernel breadth (mm), L/B: L/B Ratio, 1000 GW: 1000-grain weight, HUL: Hulling \%, MIL: Milling \%, HRR: Head rice recovery \%, B O: Bran oil \% GYP: Grain yield per plant (g). 
Table.1 Analysis of variance for yield and quality traits in rice (Oryza sativa L.)

\begin{tabular}{|c|l|c|c|c|}
\hline \multirow{2}{*}{$\begin{array}{c}\text { S. } \\
\text { No. }\end{array}$} & \multicolumn{1}{|c|}{ Character } & \multicolumn{3}{|c|}{ Mean sum of squares } \\
\cline { 3 - 5 } & $\begin{array}{c}\text { Replications } \\
(\text { d.f.=1) }\end{array}$ & $\begin{array}{c}\text { Treatments } \\
(\text { d.f.=39) }\end{array}$ & $\begin{array}{c}\text { Error } \\
(\text { d.f.=39) }\end{array}$ \\
\hline 1 & Days to flowering & 0.05 & $148.04^{* *}$ & 0.53 \\
\hline 2 & Days to maturity & 3.20 & $113.59^{* *}$ & 1.40 \\
\hline 3 & Plant height (cm) & 0.02 & $300.81^{* *}$ & 1.69 \\
\hline 4 & Number of tillers per plant & $7.32^{* *}$ & $9.70^{* *}$ & 0.22 \\
\hline 5 & Panicle length(cm) & 0.68 & $7.21^{* *}$ & 0.48 \\
\hline 6 & Number of grains per panicle & 101.29 & $7828.56^{* *}$ & 123.48 \\
\hline 7 & Kernel length (mm) & $0.04^{*}$ & $1.08^{* *}$ & 0.04 \\
\hline 8 & Kernel breadth (mm) & 0.08 & $0.09^{* *}$ & 0.05 \\
\hline 9 & L/B ratio & 0.17 & $0.46^{* *}$ & 0.01 \\
\hline 10 & Grain yield per plant (g) & 1.18 & $15.36^{* *}$ & 0.38 \\
\hline 11 & 1000 -grain weight (g) & 0.90 & $20.37^{* *}$ & 0.51 \\
\hline 12 & Hulling \% & $11.08^{* *}$ & $19.95^{* *}$ & 0.41 \\
\hline 13 & Milling \% & 0.06 & $24.82^{* *}$ & 0.50 \\
\hline 14 & Head rice recovery & $14.93^{*}$ & $47.72^{* *}$ & 1.45 \\
\hline 15 & Bran oil \% & $0.93^{*}$ & $6.40^{* *}$ & 0.20 \\
\hline$* *$ Significant at 1 per cent level * Significant at 5 per cent level \\
\hline \multicolumn{4}{|l|}{} \\
\hline
\end{tabular}

Character association studies revealed that the characters grain yield per plant showed significant and positive association with number of tillers per plant, panicle length and milling percentage. This indicated that simultaneous selection of all these characters was important for yield improvement. Similar kind of association was revealed by Satish Chandra et al., (2009), Nandan and Sweta Singh (2010) for days to flowering, Madhavilatha (2002) for days to maturity, Nandan and Sweta Singh (2010) and Ratna et al., (2015) for plant height, Idris and Mohamed (2013) for number of tillers per plant, Eswara reddy et al., (2013), Rahman et al.,(2014) for panicle length, Yadav et al., (2010) for number of grains per panicle, Reddy et al., (1997), Madhavilatha et al., (2005) for kernel length and L/B ratio (Table $3)$.

Path coefficient analysis revealed that number of tillers per plant exerted the highest positive direct effect on grain yield per plant followed by kernel breadth, L/B ratio, panicle length, milling percentage, days to flowering and head rice recovery percentage indicating that the selection for these characters was likely to bring about an overall improvement in grain yield per plant directly (Table 4). The similar results were reported by Ravindra Babu et al., (2012), Mulugeta Seyoum et al., (2012) and Imad Naseem et al., (2014).

Keeping in view of the facts, by considering all factors like per se performance, bran oil content the most promising genotypes may be utilized as parents in hybridization programme as they were expected to produce high heterotic crosses.

\section{References}

Ananth Kumar, and Verma, O.P. 2016. Genetic variability, heritability and genetic advance studies in rice (Oryza sativa L.) under sodic soil. Research in Environment and Life Sciences. 
9(2):149-151.

Anil Kumar, Jabeen, F., Cheralu, C and Devi, R.G. 2015. Correlation and path analysis of yield and yield attributing characters of rice. Bioinfolet 12 (2 B): 460-463.

Anis, G., Sabagh, A. E., Ghareb, A., Rewainy, I. E. 2016. Evaluation of promising lines in rice (Oryza sativa L.) to agronomic and genetic performance under Egyptian conditions. International Journal of Agronomy and Agricultural Research. 8(3): 52-57.

Bagati, S., Singh, A.K., Salgotra, R.K., Bhardwaj, R., Sharma, M., Rai, S.K and Bhat. 2016. Genetic variability, heritability and correlation coefficients of yield and its component traits in basmati rice (Oryza sativa L.). SABRAO Journal of Breeding and Genetics. 48 (4): 445-452.

Biswash, M.R., Sharmin, M., Rahman, N.M.F., Farhat, T and Siddique, M.A. 2016. Genetic diversity in modern $\mathrm{T}$. Aman rice varieties of Bangladesh (Oryza sativa L.). Sains Malaysiana. 45(5):709-716.

Dhurai, S.Y., Bhati, P.K and Saroj, S.K. 2014. Studies on genetic variability for yield and quality characters in rice (Oryza sativa L.) under integrated fertilizer management. An International Quarterly Journal of Life Sciences. 9 (2): 845-848.

Eswara reddy, G., Suresh, B. G and Sravan, T. 2013. Correlation and path analysis for yield and yield attributes in rice (Oryza sativa L.) genotypes. International Journal of Plant Sciences. 8 (2) : 391-394.

Ganapati, R.K., Rasul, M.G., Mian, M.A.K and Sarker, U. 2014. Genetic variability and character association of T-Aman rice (Oryza sativa L.). International Journal of Plant Biology and Research. $2(2): 1-4$.
Girish, C.T., 2015. Variability, heritability and genetic advance analysis for grain yield in rice. Journal of Engineering Research and Applications. 5 (7): 4649.

Idris, A.E., and Mohamed, K.A 2013. Estimation of genetic variability and correlation for grain yield components of rice (Oryza sativa L.). Global Journal of Plant Ecophysiology. 3 (1): 1-6.

Imad Naseem, Abdus Salam Khan and Muhammad Akhter. 2014. Correlation and path coefficient studies of some yield related traits in rice (Oryza sativa L.). International Journal of Scientific and Research Publications. 4 (4): 1-5.

Indiastat. 2015-16. Agriculture production. http: //www.indiastat.com.

Islam, M.A., Raffi, S.A., Hossain, M.A and Hasan, A.K. 2015. Analysis of genetic variability, heritability and genetic advance for yield and yield associated traits in some promising advanced lines of rice. Progressive Agriculture. 26: 2631.

Jaiswal, H.K., Srivastava, A.K and Dey, A. 2007. Variability and association studies in indigenous aromatic rice (Oryza sativa L.). Oryza. 44 (4): 351353.

Madhavilatha, L., Sekhar, M.R., Suneetha, Y and Srinivas, T. 2005. Genetic variability, correlation and path analysis for yield and quality traits in rice (Oryza sativa L.). Research on Crops. 6 (3): 527-537.

Mulugeta Seyoum, Sentayehu Alamerew and Kassahun Bantte. 2012. Genetic variability, heritability, correlation coefficient and path analysis for yield and yield related traits in upland rice (Oryza sativa L.). Journal of Plant Sciences. 7 (1): 13-22.

Nagajyothi, B., 2001. Studies on performance and heterosis of rice hybrids for yield, 
yield components and certain grain quality characters. M.Sc. (Ag.) Thesis, Acharya N.G. Ranga Agricultural University, Hyderabad.

Nandan, R., and Sweta Singh, S.K. 2010. Character association and path analysis in rice (Oryza sativa L.) genotypes. World Journal of Agricultural Sciences. 6 (2): 201-206.

Panse, V.G., and Sukhatme, P.V. 1985. Statistical methods for Agricultural workers, Indian Council of Agricultural Research, $4^{\text {th }}$ edition, New Delhi.

Rahman, M.A., Hossain, M.S., Chowdhury, I.F., Matin, M.A and Mehraj, H. 2014. Variability study of advanced fine rice with correlation, path co-efficient analysis of yield and yield contributing characters. International Journal of Applied Science and Biotechnology. 2 (3): 364-370.

Ratna, M., Begum, S., Husna, A., Dey, S.R and Hossain, M.S. 2015. Correlation and path coefficient analysis in Basmati rice. Bangladesh Journal of Agriculture Research. 40 (1): 153-161.

Ravindra Babu, V., Shreya, K., Kuldeep Singh Dangi, Usharani, $G$ and Siva Shankar, A. 2012. Correlation and path analysis studies in popular rice hybrids of India. International Journal of Scientific and Research Publications. 2 (3): 67 (1): 22-25.

Reddy, J.N., De, R.N and Suriya Rao, A.V. 1997. Correlation and path analysis in low land rice under intermediate $(0-50$ cm) water depth. Oryza. 34 (3): 187190.

Rukmini Devi, K., Parimala, K., Venkanna, V., Lingaiah, N., Hari, Y and Satish Chandra,B. 2016. Estimation of variability for grain yield and quality traits in rice (Oryza sativa L.). International Journal of Pure and Applied Bio Science. 4 (2): 250-255.

Sachan, M.S., 2015. Genetic variability and heritability studies in upland paddy in Mon district of Nagaland. Journal of Hill Agriculture. 6 (2): 269-270.

Satish Chandra, B., Dayakar Reddy, T., Ansari, N.A and Sudheer Kumar, S. 2009. Correlation and path analysis for yield and yield components in rice (Oryza sativa L.). Agricultural Science Digest. 29 (1): 45-47.

Singh, R.K., and Chaudhary, B.D. 1979. Biometrical Methods in Quantitative Genetic Analysis. Kalyani Publishers, Ludhiana.

Tara Satyavathi, C., Bharadwaj, C. H and Subramanyam, D. 2001. Variability, correlation and path analysis in rice varieties under different spacings. Indian Journal of Agricultural Research. 35 (2): 79-84.

Yadav, S.K., Suresh, B.G., Praveen Pandey and Binod Kumar. 2010. Assessment of genetic variability, correlation and path association in rice (Oryza sativa L.). Journal of Bio science. (18): 1-8.

\section{How to cite this article:}

Edukondalu, B., V. Ram Reddy, T. Shobha Rani, Ch. Aruna Kumari and Soundharya, B. 2017. Studies on Variability, Heritability, Correlation and Path Analysis for Yield, Yield Attributes in Rice (Oryza sativa L.). Int.J.Curr.Microbiol.App.Sci. 6(10): 2369-2376. doi: https://doi.org/10.20546/ijcmas.2017.610.279 